\title{
Percepção do estudante de odontologia sobre as habilidades e os fatores estressores relacionados ao atendimento odontológico infantil
}

\author{
Perception of the student of dentistry about the skills and stressors factors related to the \\ dental attendance of children
}

Yêza Teixeira de Carvalhoํ, Vinícius Lopes Marinho², Jeann Bruno Ferreira da Silva ${ }^{3}$.

\section{RESUMO}

O aluno de odontologia, tal qual o profissional, está submetido a vários fatores que são potencialmente estressantes. Recentemente, as faculdades de odontologia e seus currículos têm reconhecido que os aspectos comportamentais e emocionais dos pacientes e dos profissionais são essenciais para o resultado final do tratamento odontológico. Diante do exposto, a presente pesquisa de cunho quantitativo descritivo teve como objetivo investigar qual a percepção as principais habilidades e fatores estressores percebidos durante o atendimento infantil. Para tal fim foi aplicado um questionário fechado com acadêmicos matriculados na disciplina de Odontopediatria II no semestre de 2018/2.O questionário foi aplicado três semanas após a primeira prova da disciplina escolhida. Participaram da pesquisa 29 estudantes, sendo $21(72,4 \%)$ e $8(27,4 \%)$ dos gêneros feminino e masculino, respectivamente, com idades entre 20 e 38 anos. Apenas 8 (27,5\%) estudantes identificavam-se com a especialidade e $18(62 \%)$ gostavam de atender crianças. As principais habilidades necessárias na percepção dos estudantes foram: habilidades comunicativas $(82,7 \%)$ e conhecimento teórico das técnicas de manejo $(65,5 \%)$. Já osprincipais fatores estressores citados foram: pacientes que não aceitam tratamento $(72,4 \%)$ e manejar comportamentos não colaboradores (72,4\%). Foi possível concluir que todas as habilidades são necessárias para o atendimento odontológico infantil, sendo a mais necessária habilidade comunicativa e os principais fatores estressores relacionavam-se ao comportamento da criança.Para a análise será utilizado método quantitativo, onde será efetuada análise descritiva dos dados (frequência e porcentagem) através do software SPSS - StatisticalPackage for the Social Sciences versão 20.0 para Windows.

Palavras-chave: Atendimento infantil. Habilidades. Fatores estressores.

\section{ABSTRACT}

The student of dentistry, like the professional, is subject to several factors that are potentially stressful. Recently, dentistry faculties and their curricula have recognized that the behavioral and emotional aspects of patients and professionals are essential to the end result of dental treatment. In view of the above, the present descriptive quantitative research will aim to investigate the perception of the main skills and stressors perceived during the child care. To this end, a closed questionnaire will be applied with students enrolled in Pediatric Dentistry II in the semester of 2018/2.The questionnaire was applied three weeks after the first test of the discipline chosen. A total of 29 students participated in the study, of which $21(72.4 \%)$ and 8 (27.4\%) were male and female, respectively, aged between 20 and 38 years. Only 8 (27.5\%) students identified with the specialty and $18(62 \%)$ liked to attend children. The main skills required by the students were: communicative skills $(82.7 \%)$ and theoretical knowledge of management techniques (65.5\%). The main stressors cited were: patients who did not accept treatment $(72.4 \%)$ and manage non-cooperative behaviors $(72.4 \%)$.It was possible to conclude that all abilities are necessary for the dental care of children, being the most necessary communicative ability and the main stressors were related to the behavior of the child.Forthe analysis will be used quantitative method, where a descriptive analysis of the data (frequency and percentage) will be made through SPSS software - StatisticalPackage for the Social Sciences version 20.0 for Windows.

Keywords: child care; skills; stressors. 


\section{INTRODUÇAOAO}

O aluno de odontologia, tal qual o profissional, está submetido a vários fatores que são potencialmente estressantes. Estudos relacionam os níveis de estresse vivenciados pelos alunos com uma diminuição no seu desempenho durante a graduação. ${ }^{1}$

Recentemente, as faculdades de odontologia e seus currículos têm reconhecido que os aspectos comportamentais e emocionais dos pacientes e dos profissionais são essenciais para o resultado final do tratamento odontológico. Sabe-se que os níveis de ansiedade e estresse vivenciados pelos alunos influenciam negativamente a qualidade da aprendizagem e a execução de trabalhos, independentemente das habilidades cognitivas e técnicas. ${ }^{2}$

Um estudo para descrever e comparar o nível de estresse relatado por profissionais da área odontológica foi realizado de acordo com os autores as especialidades clínicas não diferem com o nível de estresse, porém os mais altos níveis médios foram obtidos com os odontopediatras. ${ }^{3}$

$\mathrm{O}$ estresse dos profissionais durante o exame e $\mathrm{O}$ atendimento odontopediátrico pode ser dividido em estresse produzido pela criança e aquele produzido pelo cuidador (geralmente a mãe da criança). Além disso, o estresse produzido no cirurgião-dentista tem estreita correlação com o grau de resistência ao tratamento e o tipo de comportamento. ${ }^{4}$

Diante do exposto, destaca-se a necessidade de estruturar intervenções que possam auxiliar os estudantes a enfrentar melhor o início dos trabalhos clínicos em Odontopediatria, favorecendo o desenvolvimento de habilidades para lidar com as crianças e seus acompanhantes que apresentem a elevada ansiedade, sob a perspectiva de uma formação multidisciplinar que considere a saúde na sua integralidade.

Desta maneira, questionou-se: Qual a percepção do estudante de odontologia sobre as habilidades necessárias e os fatores estressores relacionados ao atendimento odontológico infantil?

Pesquisas sobre o conhecimento da percepção do estudante de odontologia sobre as habilidades e os fatores estressores relacionados ao atendimento odontológico infantil, pode se integrar na matriz curricular algo que possa minimizar esses fatores estressores, fazendo então com que o estudante de odontologia possa realizar esse procedimento com uma maior habilidade e propriedade.

Diante disto, o presente estudo teve como objetivo investigar a percepção do 
estudante de odontologia sobre as habilidades e os fatores estressores relacionados ao atendimento odontológico infantil, bem como constatar a percepção das habilidades necessárias para atendimento infantil e identificar os fatores estressores relacionados ao atendimento.

\section{MATERIAIS E METODOS}

Trata-se de uma pesquisa de campo, quantitativo-descritiva. A pesquisa foi desenvolvida com acadêmicos matriculados na disciplina de Odontopediatria II no semestre de 2018/2 na Clínica Escola do Curso de Odontologia da Universidade de Gurupi, situado na Avenida Pará $n \cong 1544$, Centro, Gurupi-TO. Desta maneira, realizou-se um levantamento junto à coordenação do Curso e constatou-se que a quantidade de alunos matriculados no referido período eramde 42 alunos.

A escolha pela disciplina de Odontopediatria II se deu pelo fato de que nela os alunos realizam atendimento odontológico infantil, permitindo assim a investigação proposta. Utilizaram-se como critérios de inclusão: estar devidamente matriculado na disciplina de Odontopediatria II em 2018/2. Estar presente no dia da coleta de dados e aceitar a participar da pesquisa. Os critérios de exclusão foram os alunos não matriculados na disciplina de inclusão, estar ausente no dia da coleta de dados e aqueles que se recusarem á participarem da pesquisa.

A pesquisa foi submetida a um comitê de ética em pesquisa com seres humanos sob CAAE: 89299118.6.0000.5518 e aprovado conforme parecer $\mathrm{n}^{0}$ 2.691.331.

Um questionário para verificação da percepção do aluno sobre as habilidades e fatores estressores relacionados ao atendimento odontológico infantil. O questionário foi aplicado três semanas após a primeira prova da disciplina escolhida. Participaram da pesquisa 29 estudantes. Todas as etapas da pesquisa ocorreram em uma sala de uma aula da Clínica de Odontologia, devidamente preparada para tal fim.

Para a análise foi utilizado método quantitativo, descritivo dos dados (frequência e porcentagem) através do software SPSS - StatisticalPackage for the Social Sciencesversão 20.0 para Windows.

\section{RESULTADOS}

Para a obtenção dos resultados foram considerados apenas os estudantes que responderam o questionário. Do total de 42 alunos matriculados, 13 alunos entraram nos 
critérios de exclusão e 29 participaram da pesquisa realizada, sendo 21 (72,5\%) e 8 $(27,5 \%)$ estudantes do gênero feminino e masculino, respectivamente, com idades entre 20 e 38 anos.

Os dados referentes à identificação com as especialidades odontológicas em ordem decrescente obtidos em valores absolutos e percentuais, de acordo com o gênero e o total de estudantes, são apresentados na Tabela 1.

Tabela 1. Caracterização do Perfil dos Participantes.

\begin{tabular}{|c|c|c|}
\hline Variáveis & n. & $\%$ \\
\hline \multicolumn{3}{|l|}{ Sexo } \\
\hline Feminino & 21 & $72,4 \%$ \\
\hline \multicolumn{3}{|l|}{ Faixa etária } \\
\hline 26 a 30 anos & 2 & $6,8 \%$ \\
\hline Acima de 30 anos & 2 & $6,8 \%$ \\
\hline \multicolumn{3}{|c|}{ Qual(is) a(s) especialidade (s) com } \\
\hline \multicolumn{3}{|c|}{ que você mais se identifica } \\
\hline Dentística & 17 & $58,6 \%$ \\
\hline Cirurgia & 11 & $37,9 \%$ \\
\hline Endodontia & 10 & $34,4 \%$ \\
\hline Odontopediatria & 8 & $27,5 \%$ \\
\hline Periodontia & 4 & $13,7 \%$ \\
\hline Prótese & 2 & $6,8 \%$ \\
\hline \multicolumn{3}{|l|}{ Gosta de Criança } \\
\hline Sim & 25 & $86,2 \%$ \\
\hline Não & 4 & $13,7 \%$ \\
\hline \multicolumn{3}{|c|}{ Gosta de Atender Crianças } \\
\hline Sim & 18 & $62,0 \%$ \\
\hline Não & 11 & $37,9 \%$ \\
\hline
\end{tabular}

Do total de estudantes, 10 (34,5\%) responderam que se identificavam com uma única especialidade. O restante, 19 (65,5\%), responderam que se identificaram entre duas a quatro especialidades diferentes. Com relação à odontopediatria, $8(27,5 \%)$ deles 
responderam que se identificavam com a especialidade considerando que foi apenas estudantes do gênero feminino.

As justificativas citadas pelos estudantes que responderam que gostavam de atender crianças foram: "Sei lidar com crianças", "me ensinar ser mais paciente", "gosto de atende-las", "nunca teve problemas durante o atendimento", "porque tenho paciência", "porque amo crianças", "gosto do mundo lúdico", "me identifico". As justificativas dos estudantes que não gostavam de atender crianças foram: "Dão trabalho", "as crianças não colaboram", "dificuldade de fazer com que a criança aceite o tratamento", "não tenho paciência", "porque elas choram", "difícil de lidar".

Tabela 2. Valores absolutos e porcentuais das habilidades necessárias para 0 atendimento odontológico infantil do questionário de acordo com o total da amostra.

\begin{tabular}{lcc}
\hline \multicolumn{1}{c}{ Habilidades necessárias } & n. & $\%$ \\
\hline Habilidades comunicativas & 24 & $82,7 \%$ \\
$\begin{array}{l}\text { Conhecimento teórico das } \\
\text { técnicas de manejo }\end{array}$ & 19 & $65,5 \%$ \\
$\begin{array}{l}\text { Habilidades emocionais } \\
\begin{array}{l}\text { Conhecimento anatômico dos } \\
\text { dentes decíduos }\end{array}\end{array}$ & 6 & $20,6 \%$ \\
$\begin{array}{l}\text { Habilidade pratica de execução do } \\
\text { tratamento }\end{array}$ & 17 & $58,6 \%$ \\
$\begin{array}{l}\text { Habilidades anestésicas } \\
\text { Controle de tempo }\end{array}$ & 11 & $41,3 \%$ \\
$\begin{array}{l}\text { Habilidades técnicas } \\
\text { Obter uma boa coordenação }\end{array}$ & 3 & $37,9 \%$ \\
motora & 7 & $10,3 \%$ \\
$\begin{array}{l}\text { Obter um conhecimento } \\
\text { psicológico infantil }\end{array}$ & 3 & $24,1 \%$ \\
\hline
\end{tabular}

Os valores absolutos e porcentuais das habilidades necessárias obtidos no questionário de acordo com o gênero e com o total de estudantes estão apresentados na Tabela 2. Todas as habilidades necessárias listadas foram assinaladas. 
Com relação ao gênero, para o feminino, as quatro Habilidades necessárias mais assinaladas foram: habilidades comunicativas são muito importantes 18 (85,7\%); é necessário o conhecimento teórico das técnicas me manejo 12 (57,1\%); obter um conhecimento psicológico infantil 12 (57,1\%); o conhecimento anatômico dos dentes decíduos 11 (52,3\%). Para o masculino foram: É necessário o conhecimento teórico das técnicas me manejo (75,0\%); Habilidades comunicativas são muito importantes $5(62,5 \%)$; Habilidades técnicas 5 (62,5\%); O conhecimento anatômico dos dentes decíduos 4 $(50,0 \%)$.

Tabela 3. Valores absolutos e porcentuais dos fatores estressores relacionado ao atendimento odontológico infantil do questionário de acordo com o total da amostra.

\begin{tabular}{|c|c|c|}
\hline $\begin{array}{c}\text { Fator } \\
\text { Estressor }\end{array}$ & n. & $\%$ \\
\hline Lidar com as expectativas dos pacientes; & 2 & $6,8 \%$ \\
\hline $\begin{array}{l}\text { Lidar com as expectativas dos pais dos } \\
\text { pacientes; }\end{array}$ & 5 & $17,2 \%$ \\
\hline Relacionar-se com os pacientes; & 7 & $24,1 \%$ \\
\hline Relacionar-se com os pais dos pacientes; & 2 & $6,8 \%$ \\
\hline Pacientes que não aceitam o tratamento; & 21 & $72,4 \%$ \\
\hline Lidar com a dor e a ansiedade do paciente; & 18 & $62,0 \%$ \\
\hline Lidar com a ansiedade dos pais; & 4 & $13,7 \%$ \\
\hline Manejar comportamentos não colaboradores; & 21 & $72,4 \%$ \\
\hline $\begin{array}{l}\text { Lidar com os problemas das faltas, } \\
\text { cancelamentos e atrasos; }\end{array}$ & 15 & $51,7 \%$ \\
\hline Pacientes que não seguem instruções; & 11 & $37,9 \%$ \\
\hline Pais que não seguem instruções. & 13 & $44,8 \%$ \\
\hline
\end{tabular}


Os valores absolutos e porcentuais dos fatores estressores obtidos no questionário de acordo com o gênero e com o total de estudantes estão apresentados na Tabela 3. Todos os fatores estressores listados foram assinalados.

Com relação ao gênero, para o feminino, os quatro fatores estressores mais assinalados foram: manejar comportamentos não colaboradores 16 (76,1\%); pacientes que não aceitam o tratamento 14 (66,6\%); lidar com a dor e a ansiedade do paciente 11 $(52,3 \%)$; lidar com os problemas das faltas, cancelamentos e atrasos $11(52,3 \%)$. Para o masculino foram: pacientes que não aceitam o tratamento 6 (75,0\%); manejar comportamentos não colaboradores 6 (75,0\%); lidar com a dor e a ansiedade do paciente 5 (62,5\%); pacientes que não seguem instruções $4(50,0 \%)$.

A odontologia é a profissão considerada uma das mais estressantes da área da saúde, e na odontopediatria, além dos fatores relacionados ao paciente, devem ser considerados também os pais e/ou responsáveis.5. Sendo então necessário obter habilidades para o atendimento odontológico infantil. Sendo assim, o estudo teve como objetivo avaliar a percepção do estudante de odontologia sobre as habilidades e os fatores estressores relacionados ao atendimento odontológico infantil. ${ }^{5}$

Foi utilizado um questionário, por ser um método rápido, ter baixo custo, por dar maior grau de liberdade e tempo ao participante, além da possibilidade de menores distorções por permitir a obtenção de dados muitas vezes superficiais e os dados detalhados poderem ser obtidos com as questões abertas. ${ }^{6}$

\section{DISCUSSĀO}

Embora os estudantes tenham contato com crianças em outras disciplinas, é na odontopediatria 1 que eles têm a oportunidade de aprender como aplicar técnicas de gerenciamento comportamental e os procedimentos direcionados especificamente para crianças. Sendo assim, a amostra selecionada foi de estudantes matriculados na disciplina de odontopediatria 1, que é a primeira disciplina de odontopediatria ministrada no curso de graduação em odontologia da Universidade de Gurupi.

As mulheres são maioria entre os ingressantes e concluintes, indicando a tendência de aumento do predomínio feminino nos cursos de odontologia. Sendo então de acordo com os dados da presente pesquisa, onde a grande maioria dos participantes pertencia ao gênero feminino.Com relação à especialidade, apenas uma pequena porcentagem da amostra identificava-se com a odontopediatria; a maioria pertencia ao gênero feminino. 
No plano nacional, entre as especialidades que possuem franca maioria feminina, está, em primeiro lugar, a odontopediatria (85,0\%). Apesar de atualmente ser uma profissão predominantemente de mulheres, algumas áreas ainda são consideradas masculinas como a Cirurgia Bucomaxilofacial, enquanto outras como a Odontopediatria e a Dentística áreas femininas. No caso específico da Odontopediatria, sugere-se, também, que a maior participação feminina na composição da força de trabalho esteja relacionada a aspetos comportamentais culturalmente associados às mulheres, tais como o ideal de serviço social e a responsabilidade - derivada da maternidade - pelo cuidado infantil. ${ }^{78.9}$

Outros estudos concluíram que estas situações podem estar ligadas a uma tendência de diferenças em características entre os gêneros dos profissionais. Uma destas diferenças é que os dentistas do gênero masculino tendem a utilizar mais manobras aversivas do que os do gênero feminino para o controle do comportamento. ${ }^{10}$.

As justificativas dos acadêmicos que não gostavam de atender crianças foram justamente relacionadas ao comportamento da mesma. As justificativas dos Acadêmicos também demonstram uma relação direta com os fatores estressores mais citados nos dois questionários, pois estes fatores foram justamente os pacientes que não aceitaram tratamento e manejaram tratamentos não colaboradores. Alguns estudos relatam que 0 estudante de odontologia está sob muitos potenciais fatores de estresse, incluindo o relacionamento com pacientes que não colaboram, não aceitam tratamento ou estão muito ansiosos em relação ao tratamento. ${ }^{2,3}$

É valido afirmar que o nível de estresse vivenciado pelos estudantes influencia negativamente a qualidade e a execução do trabalho clínico, independentemente das habilidades cognitivas e técnicas ${ }^{5}$

O estudante de odontologia, assim como o profissional da odontologia, é submetido a vários fatores potencialmente estressantes. $O$ tratamento odontológico de uma criança requer não apenas a preparação técnica, mas também o conhecimento e a capacidade de lidar com essa fase do desenvolvimento, utilizando técnicas especiais de gerenciamento. ${ }^{2}$

\section{CONSIDERAÇOEES FINAIS}

Após a análise dos resultados obtidos na presente pesquisa pôde se concluir que todas as habilidades são necessárias, sendo consideradas pelos estudantes a mais importante áhabilidade comunicativa para o atendimento odontopediatrico. Em relação aos fatores estressores associados ao estresse durante o atendimento, todos os fatores 
relacionados ao paciente infantil foram considerados estressores, principalmente fatores associados ao comportamento da criança.

Houve limitações para realização da pesquisa, pois não foi possível a comparação direta dos resultados deste estudo com outros, porque não foi encontrado estudos semelhantes na literatura. Deixando então a sugestão de novas pesquisas abordando o tema, visto que a atuação em odontopediatria ainda é um grande desafio para boa parte dos profissionais.

\section{REFERÉNCIAS}

1. Macchi, R.; Biondi, A. M.; Cortese, S. G. Influencias de variables em lacalidaddeldesempeño clínico em alumnos de lacátedra de odontología integral niños. Revista de la FacultadOdontología. 1996, Jul.(no 41): 35-40.

2. Cardoso C.I.,Loureiro Sr.; Nelson-Filho, P. Pediatric dental treatment: manifestationsof stress in patients, mothersand dental schoolstudents.Braz Oral Res. 2004 (n2):150-5.

3. Newton, J.T.; Mistry, K.;Patel A.; Perkins, M.; Saeed, K.; Smith, C. Stress in dental specialists: a comparison of six clinical dental specialties.Prim Dent Care. 2002, $\operatorname{Jul}\left(n^{\circ}-3\right): 100-4$.

4. Kan, M.; Ishikawa, T.; Nagasaka, N.A study of psychological stress created in dentists by childrenduring pediatric dental treatment.Journal of Dentistry for Children. 1999, Jun (no 1): 41-8.

5. Vargas, A.N.; Fontes, B. N. V.; Tolentino, A. B.; Rodrigues, L. C. M.; Oliveira, F. S.; Castro, A. M.Percepção do estudante de Odontologia sobre os fatores estressores relacionados ao atendimento infantil. Faculdade de Odontologia de Lins/Unimep. 2013, Jan ( $\mathrm{n}^{\circ}$ 1): 13.

6. Cunha M.B.;Metodologias para estudo de usuários de informação científica e tecnológica. Revista Biblioteconomia de Brasilia. 1982, Out (no 2):5-19.

7. Morita M.C.; Haddad A, Araújo Me. Perfil atual e tendências do cirurgião-dentista brasileiro. (Ed. № 1)Maringuá. Dental Press International; 2010.

8. Baldissera, R.S.; Grecca, F.S.; Santos, R.B. Participação das Mulheres na Graduação da Faculdade de Odontologia da Universidade Federal do Rio Grande do Sul. Rev. Fac. Odontol. 2010, Jan (V. 51, n. 1): p. 27-30.

9. Arouca, R.; Levy, S.C.; Perfil demográfico da força de trabalho em odontopediatria no Estado do Rio de Janeiro. Rev. bras. odontol. 2010 Jul. (V. 67, n. 2): p.178-82.

10. Barbabela, D.; Mota, J.B.T.; Maia, P. G. M.; Bonanato, K.; Paiva, S. M.; Pordeus, I. A. Preferência da criança pelo gênero do odontopediatra.Arquivos em Odontologia.2008, Abr(V. 44 № 2). 